\title{
Double Y-stenting for tracheobronchial stenosis
}

\author{
Masahide Oki and Hideo Saka
}

ABSTRACT: The purpose of the present study was to evaluate the feasibility, efficacy and safety of the double Y-stenting technique, by which silicone Y-stents are placed on both the main carina and another peripheral carina, for patients with tracheobronchial stenosis.

Under general anaesthesia, using rigid and flexible bronchoscopes, a Dumon ${ }^{\mathrm{TM}} \mathrm{Y}_{\text {-stent }}$ (Novatech, La Ciotat, France) was first placed on the primary right or secondary left carina followed by another Y-stent on the main carina so as to insert the bronchial limb of the stent into the first Y-stent. Patients who underwent double Y-stent placement during 3 yrs and 1 month in a single centre were retrospectively reviewed.

In the study period, 93 patients underwent silicone stent placement and 12 (13\%) underwent double Y-stent placement (11 for right and one for left bronchus). A combination of Y-stents, $14 \times 10 \times 10 \mathrm{~mm}$ and $16 \times 13 \times 13 \mathrm{~mm}$ in outer diameter, were most frequently used. Dyspnoea was relieved in all patients. Six out of seven patients with supplemental oxygen before stent placement could be discharged without supplemental oxygen. Median survival after stenting was 94.5 days. One pneumothorax and one granuloma formation occurred.

Double Y-stent placement for patients with tracheobronchial stenosis was technically feasible, effective and acceptably safe.

KEYWORDS: Airway stenosis, double Y-stenting, silicone, stent, Y-stent

0 ince central airway stenosis is often a lifethreatening condition, prompt treatment is required. Airway stenting for patients with central airway stenosis is a well-established procedure to relieve severe respiratory symptoms immediately [1-5]. Ideally, the length of the stent should be minimised while covering the whole stenotic region and the shape of the stent should be chosen to fit the anatomical structure of the airway. Of the two main types of airway stents, silicone and metal, silicone stents can be easily customised on-site with cutting or combining to fit the individual stenotic airway [6].

For stenosis around the main carina, bifurcated stents, so-called "Y-stents," have been used [7-9]. However, we often encounter patients with airway stenosis ranging from the trachea to the orifice of the lobar bronchi, which cannot be wholly covered with a single Y-stent. For such cases, we have performed double Y-stenting in which silicone Y-stents are placed on both the main carina and another peripheral carina, such as the one between the bronchus to the right, upper lobe and the bronchus intermedius (primary right carina (RC1)) or the carina between the bronchus to the lingular segment of the left upper-lobe and the left lower-lobe bronchus (secondary left carina (LC2)) [10]. To our knowledge, no data have, so far, been reported on this double Y-stenting procedure, except our case reports focusing on the technical details [10]. In this retrospective study, we investigated the feasibility, efficacy and safety of the double Y-stenting technique for patients with tracheobronchial stenosis.

\section{METHODS}

\section{Patients}

From January 2008 to February 2011, we performed 107 silicone stenting procedures for 93 patients with airway stenosis, obstruction or fistula at Nagoya Medical Center (Nagoya, Japan). Among the patients, 12 underwent double Y-stenting using Dumon $^{\mathrm{TM}}$ Y-stents (Novatech, La Ciotat, France). The medical records of these 12 patients were retrospectively reviewed. Prior to stent placement, written informed consent for the procedure was obtained from all the patients and/or their families. The institutional review board of Nagoya Medical Center approved this retrospective study (identifier 2011-437). Some of these patients were previously reported as case reports focused on the stent insertion technique [10].

\section{AFFILIATIONS}

Dept of Respiratory Medicine Nagoya Medical Center, Nagoya, Japan.

CORRESPONDENCE

M. OKi

Dept of Respiratory Medicine Nagoya Medical Center 4-1-1 Sannomaru

Naka-ku

Nagoya 460-0001

Japan

E-mail: masahideo@aol.com

Received:

Jan 252012

Accepted after revision:

Feb 292012

First published online:

April 102012 


\section{Procedures}

All procedures were performed using rigid and flexible bronchoscopes under general anaesthesia. After bronchoscopic airway re-establishment was performed using tools and devices such as argon plasma coagulation, electrocautery, high-pressure balloon or the bevel of the rigid bronchoscope, we performed double Y-stenting using Dumon stents in which a peripheral Ystent was placed on RC1 or LC2 followed by a proximal Y-stent on the main carina, in the manner described in Proximal Y-stent placement section. After the stent insertion, saline solution and/ or acetylcysteine nebulisation were performed three times a day. Double Y-stenting was indicated in cases with airway stenoses or invasion around both the main carina and RC1 or LC2. Cases where the bronchus beyond the segmental bronchus was invaded and obstructed (e.g. cases with the tumour extending from the peripheral bronchus) were revealed by flexible bronchoscopes [11], and we did not consider as having indications for stenting.
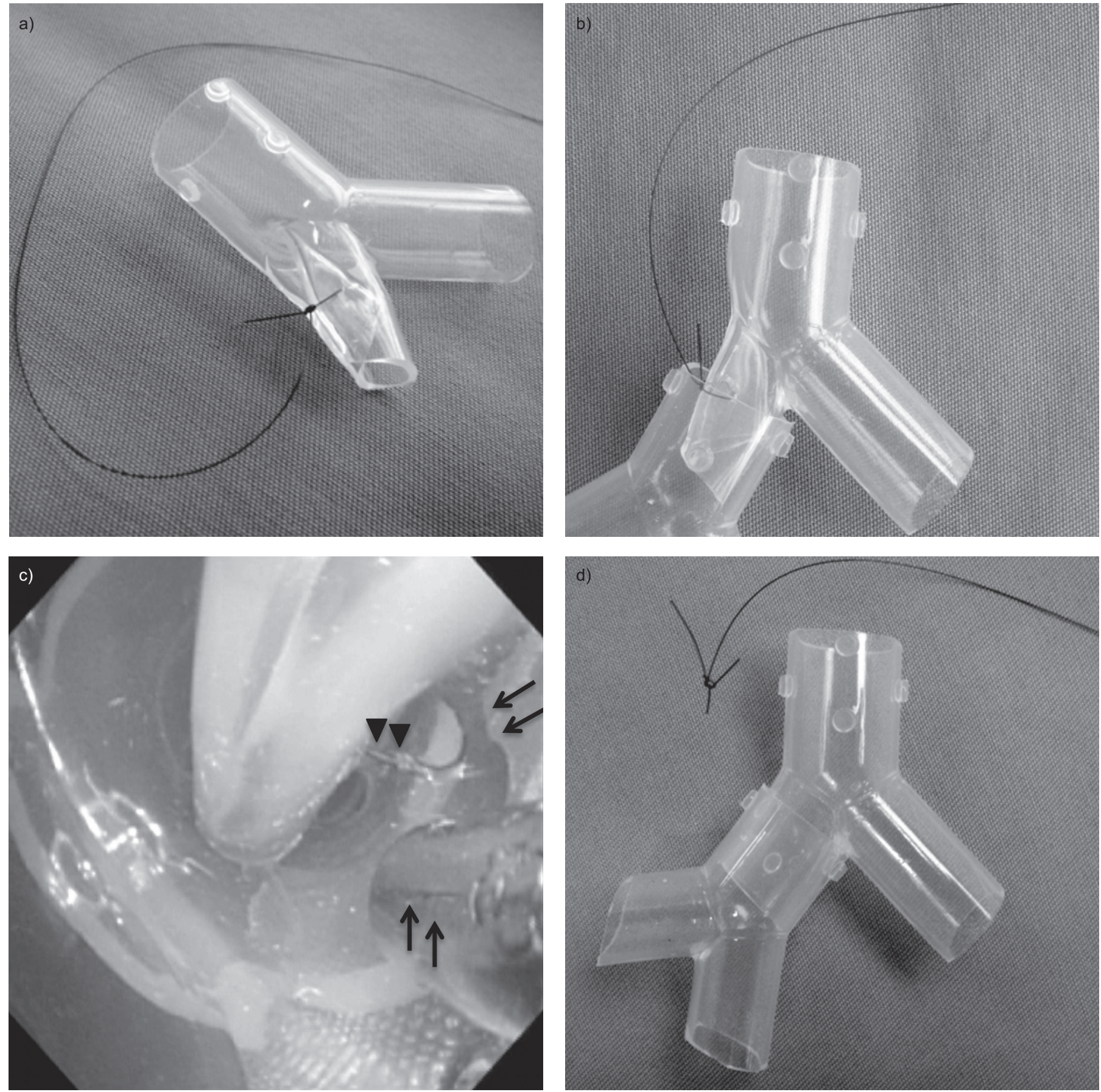

FIGURE 1. a) Peripheral Y-stent and proximal Y-stent with limb folded and tied with nylon thread to maintain folded shape. b) Folded limb of proximal Y-stent inserted into peripheral Y-stent. c) Endoscopic flexible scissors (arrows) about to cut nylon thread (arrowheads). d) Assembled Y-stents after nylon thread removal. 


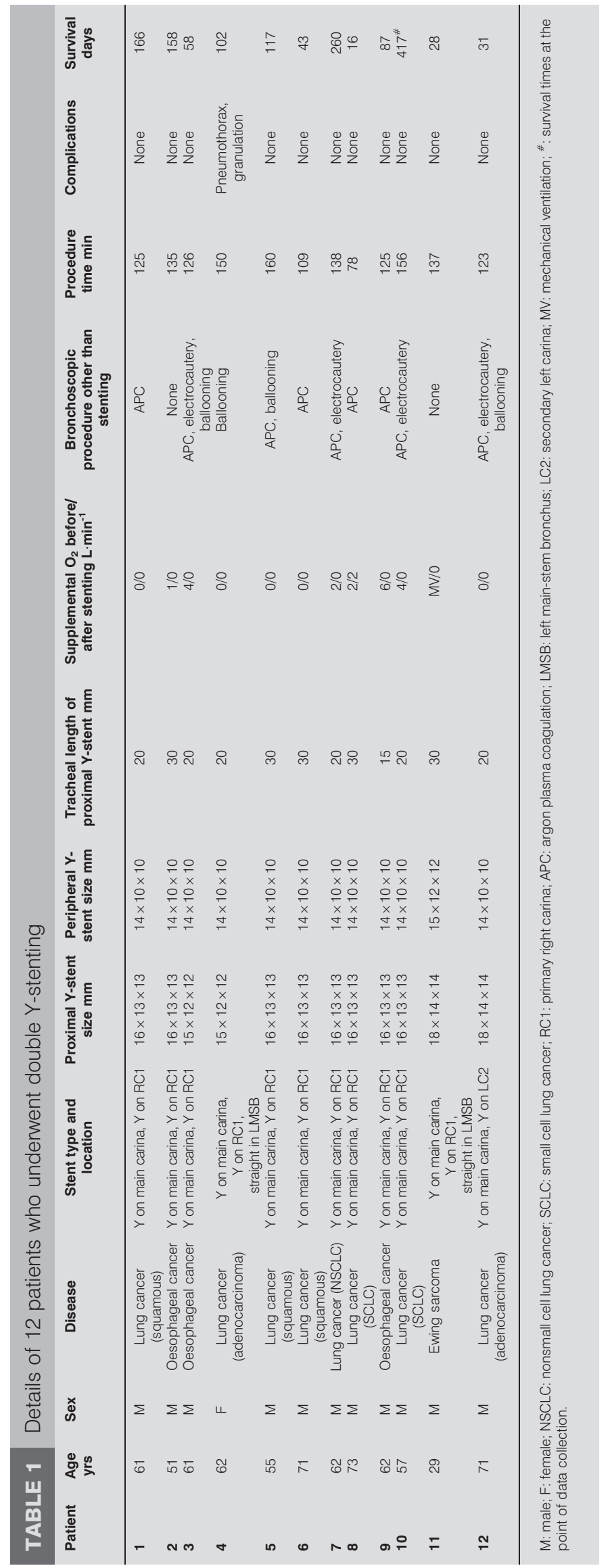

\section{Peripheral Y-stent placement}

Dumon Y-stents are available in both non-radiopaque and radiopaque silicone. Either can be used in this technique, and the latter can be inserted under fluoroscopic guidance. Dumon Y-stents are available in four different outer diameters: $14 \times 10 \times 10 \mathrm{~mm}, 15 \times 12 \times 12 \mathrm{~mm}, 16 \times 13 \times 13 \mathrm{~mm}$ and $18 \times 14 \times 14 \mathrm{~mm}$. The size and length of the stent are decided using a flexible bronchoscope, endobronchial ultrasound with a balloon and pre-procedural computed tomography as previously described [12]. The Y-stents are designed to fit the anatomical structure of bronchi around the main carina, and so the left limb of the Y-stent branches off at an acute angle from the tracheal limb compared with the right limb. Hence, when the Dumon Y-stent is used as a peripheral Y-stent on RC1, the left limb of the Y-stent should be inserted into the right upper lobe bronchus and the right limb should be inserted into the bronchus intermedius. Additionally, the bronchial orifice should be cut at an angle so as not to face the bronchial wall. A peripheral Y-stent is placed so as to straddle the RC1 or LC2 using the following push or pull method [9].

\section{Push method}

The Y-stent is inserted into the right/left main stem bronchus just above RC1/LC2. Then the stent is grasped with a rigid forceps and pushed so as to straddle the bifurcation.

\section{Pull method}

This technique can be used only for right bronchial stenosis because of the absence of the bronchus intermedius in the left bronchus. Before stent insertion, the limb of the Y-stent for the right upper-lobe bronchus is cut shorter than the other limb for the bronchus intermedius. First, both limbs of the Y-stent are inserted into the bronchus intermedius. Then, the stent is grasped with the rigid forceps and slowly pulled back until a limb slips into the right upper lobe bronchus. Finally, the stent is pushed to fit on the bifurcation.

\section{Proximal Y-stent placement}

A proximal Y-stent is placed so as to insert the right or left limb into the peripheral Y-stent. For good attachment of the peripheral and proximal Y-stents, a Y-stent, in which the outer diameter of bronchial limb is equal to or slightly larger than the inner diameter of the peripheral Y-stent, should be selected for the proximal stent. Additionally, the tracheal length of the proximal Y-stent should be minimal in order to facilitate mucus discharge. Before insertion, the bronchial limb of the proximal Y-stent for inserting into the peripheral Y-stent is folded and tied with a nylon thread to keep the folded shape (fig. 1a). The Y-stent is then loaded into the stent introducer tube while the end of the nylon thread protrudes to the outside through the stent introducer tube for easy removal. The stent insertion is performed with a standard Y-stenting technique using a push or pull method with/without fluoroscopic guidance. Once the folded limb has been inserted into the peripheral Y-stent (fig. 1b), the tied thread is cut with endoscopic flexible scissors under flexible bronchoscopic control (fig. 1c) and removed. The folded limb expands automatically or by using rigid forceps or a high-pressure balloon (fig. 1d). 

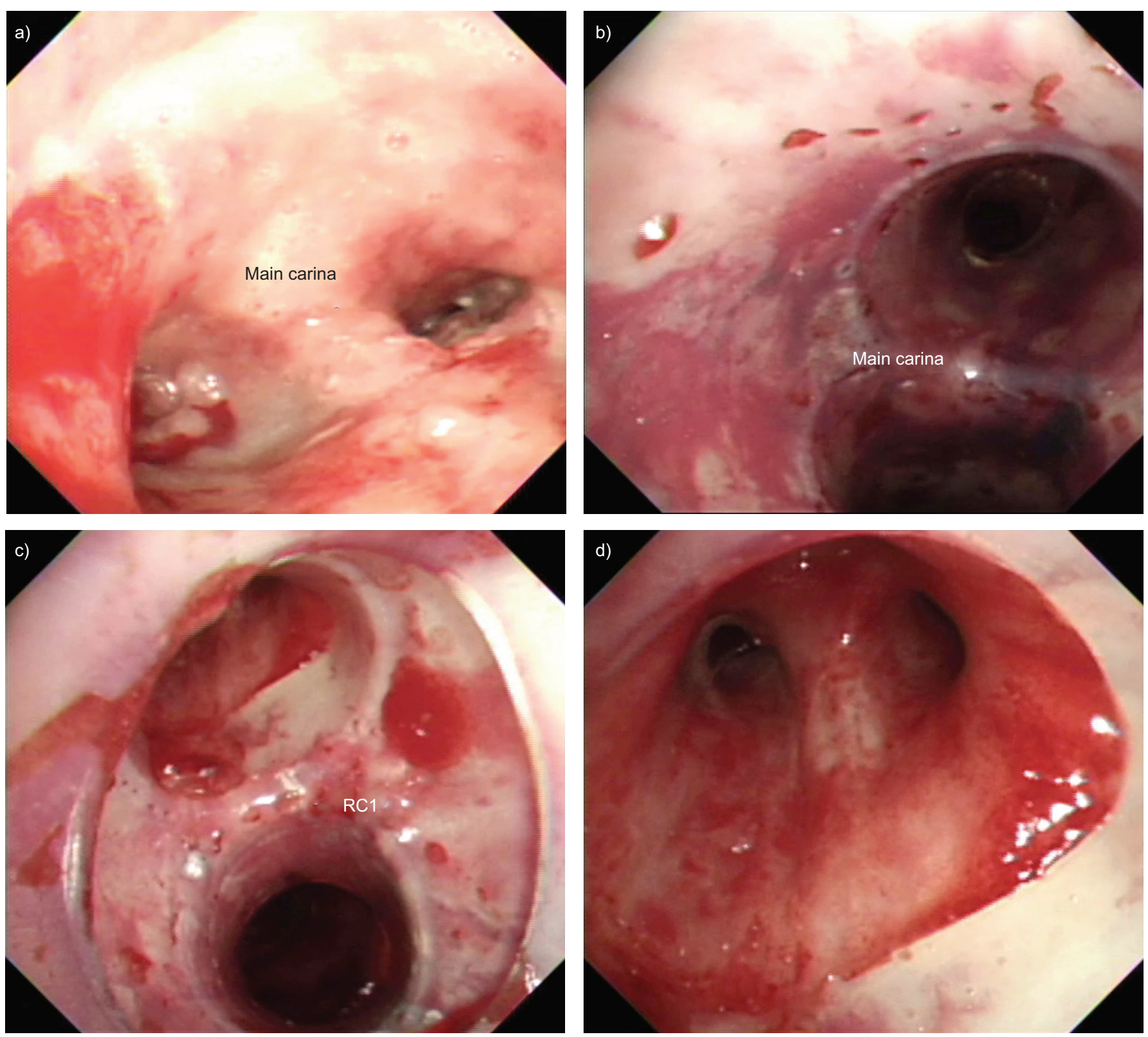

FIGURE 2. Bronchoscopic views showing main carina invaded by oesophageal cancer a) before stent placement, b) after double Y-stent placement using nonradiopaque Dumon Y-stents, c) at the joint of the proximal and peripheral Y-stents and d) at the distal end of peripheral Y-stent in right upper lobe bronchus. RC1: primary right carina.

\section{RESULTS}

\section{Patients}

During the study period, we performed double Y-stent placement for 12 patients (13\% of patients with silicone stent placement, 11 males and one female (mean age of 59.5 yrs, range 29-73 yrs). The details are shown in table 1. All patients had malignant disease with tracheobronchial stenosis; nonsmall cell lung cancer in six, small cell lung cancer in two, oesophageal cancer in three and Ewing sarcoma in one. Dyspnoea was relieved more or less in all patients $(100 \%)$, based on their medical and stenting records. Of seven patients with supplemental oxygen before stenting, six $(86 \%)$ patients including one who was mechanically ventilated, could be discharged without supplemental oxygen. The stents could be removed in one patient with small cell lung cancer because of tumour response to chemotherapy. Median survival after the procedure at the time of data collection was 94.5 days (range 16-417 days).

\section{Procedures}

11 of the 12 patients underwent double Y-stent placement in the right bronchus and the remaining patient in the left bronchus. In four patients, insertion of the stent limb into the right upper-lobe bronchus was difficult, and so the insertion was performed using the same technique with a nylon thread that was used to insert the limb of the proximal Y-stent into the peripheral Y-stent. A combination of Y-stents, $14 \times 10 \times 10 \mathrm{~mm}$ and $16 \times 13 \times 13 \mathrm{~mm}$ in 

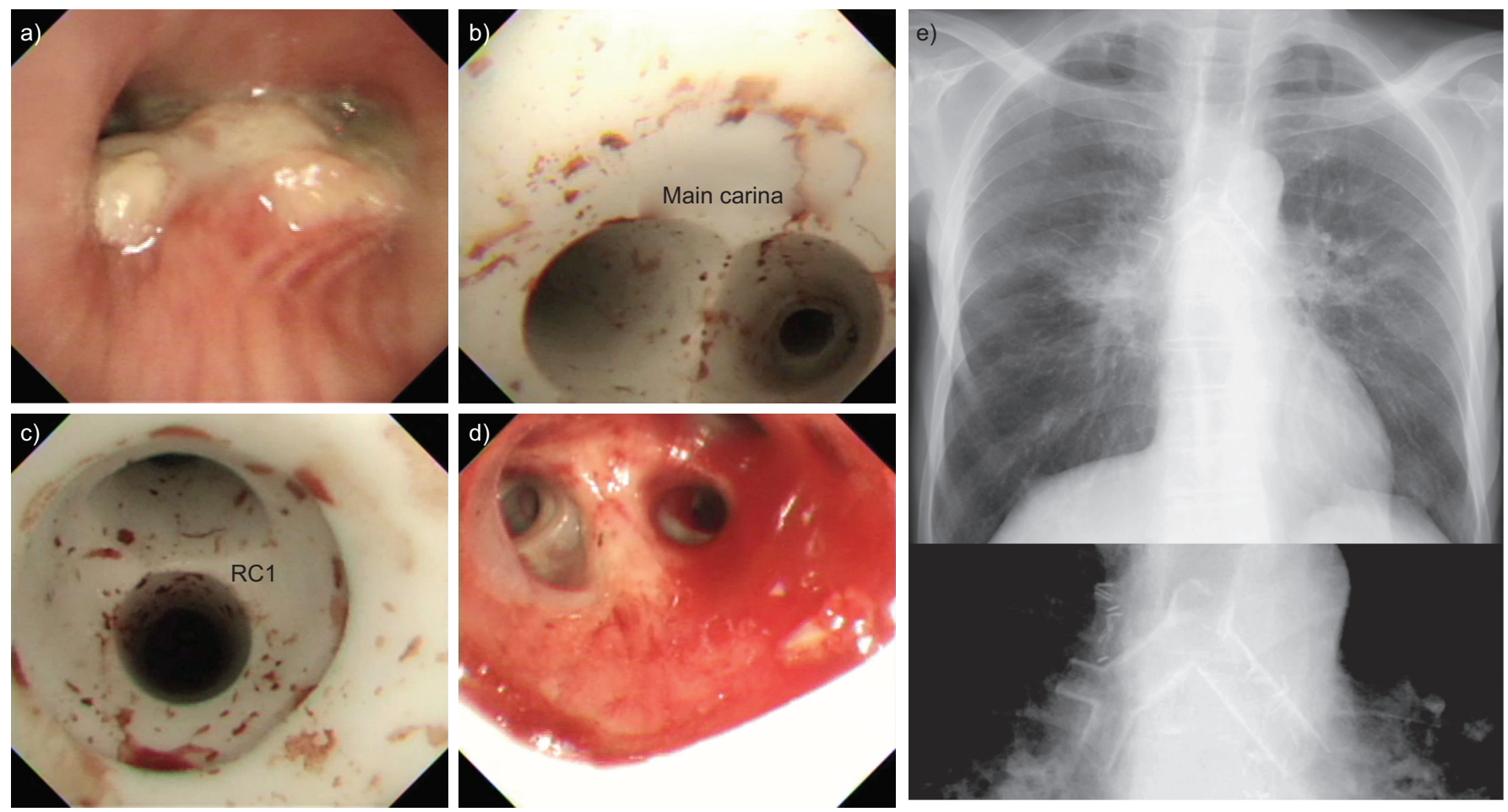

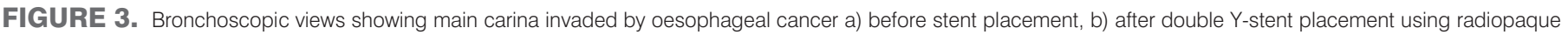

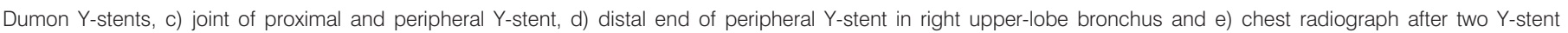
placements. $\mathrm{RC} 1$ : primary right carina.

outer diameter, was most frequently used (eight patients). A radiopaque stent and a non-radiopaque stent were used in seven and five patients, respectively. Mean procedure time for double Y-stenting was $130 \mathrm{~min}$ (range 78-160 min). Representative patients ( 3 and 9 in table 1 ) are shown in figures 2 and 3 , respectively.

\section{Safety}

One patient developed pneumothorax, which was revealed by chest radiograph just after the procedure. It healed spontaneously without drainage. The patient also developed the obstruction at the peripheral end of the Y-stent due to granulation tissue formation that required bronchoscopic resection using argon plasma coagulation 7 weeks after the procedure.

\section{DISCUSSION}

Currently, there are a variety of stents available in different materials, shapes and sizes [2-5]. Silicone stents, developed by DUMON [13], in which mainly the straight and Y-shape stents are available [8], have been widely used in the treatment of airway stenosis, and their efficacy and safety are well-established [14]. The stents can be customised with cutting or in combination for each stenotic airway, and so they are applicable in most cases with central airway stenosis [6]. However, despite the flexible utility of a silicone stent, stenting of the upper lobe bronchus, which a rigid bronchoscope cannot reach directly, has posed a challenging problem. Conventionally, a fenestration method has been used, in which a window is cut in the wall of the stent crossing the orifice of the upper lobe bronchus to allow ventilation of the upper lobe lung [6]. However, though it can aerate the upper lobe lung temporarily, the window does not maintain the upper-lobe airway patency. Additionally, the tumour or granulation tissue is likely to protrude into the stent lumen from the window. To overcome these drawbacks, we reported a technique in which a Dumon Y-stent was placed in RC1 [12]. Afterward, we developed the double Y-stenting technique so as to treat the stenotic airway around both the main carina and RC1 [10]. Double Y-stenting relieved dyspnoea in all cases and six of the seven cases with supplemental oxygen, including one patient requiring mechanical ventilation, were able to discontinue supplemental oxygen. These findings suggest the clinical efficacy of the stenting technique.

As described in the Methods section, we used a technique in which the bronchial limb of the Y-stent was sewed and tied with a nylon thread to maintain the folded shape for inserting the limb of the proximal Y-stent into the peripheral Y-stent. This technique has made it possible to insert the limb of the Y-stent into the narrow lumen. Using this technique the limb of the proximal Y-stent, with an equal or even slightly larger outer diameter compared with the inner diameter of the peripheral $Y$ stent, could be inserted into the peripheral Y-stent. Actually, in most of our cases, the bronchial limb with an outer diameter of $13 \mathrm{~mm}$ could be inserted successfully into the peripheral stent with an inner diameter of $12 \mathrm{~mm}$. As a result, both stents fit into each other well without a gap, which minimised retention of secretion or tumour invasion. Additionally, this technique was used in four cases presenting technical difficulties in order to insert the limb of the peripheral Y-stent into the right upper lobe bronchus. The double Y-stenting technique is indeed more complicated than the conventional Y-stenting technique. Naturally, the procedural time of the Y-stenting technique was 
longer than the conventional single Y-stenting technique (mean 130 min versus 55 min, respectively; calculated from the data of 37 patients who underwent single Y-stent placement during the study period). However, we did not perform stenting using the conventional fenestration method for aerating the upper lobe lung in any cases during the study period. This would suggest the impressively high feasibility of the double Y-stenting technique in experienced hands.

In the largest reported study of 1,058 patients with Dumon stent placement [14], the frequent complications related to silicone stenting were reported to be migration $(9.5 \%)$, granulation $(7.9 \%)$ and obstruction by secretions (3.6\%). In a study of 86 patients who underwent Dumon Y-stent placement [9], only two (one severe cough and one migration) stent-related complications were observed. The potential complications associated with double Y-stenting could be similar to those of other silicone stenting procedures. Above all, retention of secretion might be a possible adverse effect. However, the management of mucus retention similar to patients with single Y-stent placement seems to be sufficient for patients with double Y-stent placement. In our institution, we manage patients with regular nebulisation for as long as possible after stenting with the double Y-stenting method as well as other methods, such as single Y-stenting and straight-stenting. However, we do not routinely perform other special management, such as bronchodilator therapy or regular bronchoscopy. It is well known that stent length influences the occurrence of mucus retention. In our cases, the length of the tracheal limb of the proximal Y-stent was much shorter (mean (range) length 23.8 (15-30) $\mathrm{mm}$ than the tracheal limb of the Y-stent in patients with single Y-stenting during the study period ( $\mathrm{n}=37 ; 39.9$ (5-90) mm). Hence, the total length of stents in the double Y-stenting technique in our cases was not much longer than that in the conventional single Y-stenting technique we frequently use in our daily practice. The short tracheal limb in our cases may well have prevented obstruction by secretions.

During the study period, the double Y-stent placement was performed in $13 \%$ of all patients who underwent silicone stent placements. The frequency of patients with the indication was unexpectedly high. As described, now available silicone Y-stents can be placed on RC1. However, the insertion sometimes requires thread tie-back to keep the folded shape or cutting the orifice of the stent at an angle so as not to face the bronchial wall. Additionally, Y-stents which were designed to fit the main carina might be too large for the stenosis around RC1 in some cases. We are now developing dedicated bifurcated stents for RC1 with exclusive limb angles or sizes, for greater technical feasibility.

\section{Conclusions}

Double Y-stent placement for patients with airway stenosis around both the main carina and RC1 or LC2 was technically feasible, effective and acceptably safe.

\section{STATEMENT OF INTEREST}

None declared

\section{REFERENCES}

1 Seijo LM, Sterman DH. Interventional pulmonology. N Engl J Med 2001; 344: 740-749.

2 Ernst A, Feller-Kopman D, Becker HD, et al. Central airway obstruction. Am J Respir Crit Care Med 2004; 169: 1278-1297.

3 Bolliger CT, Sutedja TG, Strausz J, et al. Therapeutic bronchoscopy with immediate effect: laser, electrocautery, argon plasma coagulation and stents. Eur Respir J 2006; 27: 1258-1271.

4 Freitag L. Airway stents. Eur Respir Monogr 2010; 48: 190-217.

5 Lee P, Kupeli E, Mehta AC. Airway stents. Clin Chest Med 2010; 31: 141-150.

6 Breen DP, Dutau H. On-site customization of silicone stents: towards optimal palliation of complex airway conditions. Respiration 2009; 77: 447-453.

7 Freitag L, Eicker R, Linz B, et al. Theoretical and experimental basis for the development of a dynamic airway stent. Eur Respir J 1994; 7: 2038-2045.

8 Dumon JF, Dumon MC. Dumon-Novatech Y-stents: four-year experience with 50 tracheobronchial tumors involving the carina. J Bronchol 2000; 7: 26-32.

9 Dutau H, Toutblanc B, Lamb C, et al. Use of the Dumon Y-stent in the management of malignant disease involving the carina: a retrospective review of 86 patients. Chest 2004; 126: 951-958.

10 Oki M, Saka H, Kitagawa C, et al. Double Y-stent placement for tracheobronchial stenosis. Respiration 2010; 79: 245-249.

11 Oki M, Saka H. Thin bronchoscope for evaluating the stenotic airway during stenting procedure. Respiration 2011; 82: 509-514.

12 Oki M, Saka H, Kitagawa C, et al. Silicone Y-stent placement on the carina between bronchus to the right upper lobe and bronchus intermedius. Ann Thorac Surg 2009; 87: 971-974.

13 Dumon JF. A dedicated tracheobronchial stent. Chest 1990; 97: 328-332.

14 Dumon JF, Cavariere S, Diaz-Jimenez JP, et al. Seven-year experience with the Dumon prosthesis. J Bronchol 1996; 3: 6-10. 